\title{
A Geometric Preferential Attachment Model of Networks II
}

\author{
Abraham D. Flaxman*, Alan M. Friezeł Juan Vera
}

March 19, 2008

\begin{abstract}
We study a random graph $G_{n}$ that combines certain aspects of geometric random graphs and preferential attachment graphs. This model yields a graph with power-law degree distribution where the expansion property depends on a tunable parameter of the model.

The vertices of $G_{n}$ are $n$ sequentially generated points $x_{1}, x_{2}, \ldots, x_{n}$ chosen uniformly at random from the unit sphere in $\boldsymbol{R}^{3}$. After generating $x_{t}$, we randomly connect it to $m$ points from those points in $x_{1}, x_{2}, \ldots, x_{t-1}$.
\end{abstract}

\section{Introduction}

During the last decade a large body of research has centered on understanding and modeling the structure of large-scale networks like the Internet and the World Wide Web. Several recent books provide a general introduction to this topic [38, 41]. One important feature identified in early experimental studies (including $[4,13,23]$ ) is that the vertex degree distribution of many real-world networks has a heavy-tailed property, which may follow a power-law (i.e., the proportion of vertices of degree at least $k$ is proportional to $k^{-\alpha}$ for some constant $\alpha$ ). This has driven the investigation of random graph models which generate heavy-tailed degree distributions, including the fixed degree sequence model, the copying model, and the preferential attachment model.

The preferential attachment model and its derivatives have been particularly popular for theoretical analysis. Preferential attachment was proposed as a model for real-world complex networks by Barabási and Albert [5]. The distribution was formalized by Bollobás and Riordan [10], and in [12] it was proved rigorously that whp a graph chosen according to this distribution has a power-law degree distribution with complementary cumulative distribution function (ccdf) $\operatorname{Pr}[\operatorname{deg}(v) \geq k]=\Theta\left(k^{-2}\right)$. By changing the initial attactiveness or extending allowable graph operations, the power of the ccdf power-law can be tuned to take any value in the interval $(1, \infty)[14,18]$.

However, there are some significant differences between graphs generated by preferential attachment and those found in the real world. One major difference is found in

\footnotetext{
${ }^{*}$ Microsoft Research

${ }^{\dagger}$ Carnegie Mellon University; supported in part by NSF grant CCF-200945.

${ }^{\ddagger}$ Georgia Institute of Technology
} 
their expansion properties. Mihail, Papadimitriou, and Saberi [36] showed that whp the preferential attachment model has conductance bounded below by a constant. On the other hand, Blandford, Blelloch and Kash [8] found that some WWW related graphs have smaller separators than the preferential attachment model predicts. This observation is consistent with observations due to Estrada [20], who found that half of the real-world networks he looked at were good expanders and the other half were not so good. The perturbed random graph framework provides one approach to understanding expansion in real-world networks [24], but it does not give a generative procedure. This paper investigates a generative procedure, based on a geometric modification of the preferential attachment model, which yields a graph that might or might not be a good expander, depending on a tunable parameter of the geometry. This is a strict generalization of the geometric preferential attachment graph developed in [25] which was designed specifically to avoid being a good expander.

The primary contribution of this paper is to provide a parameterised model that exhibits a sharp transition between low and high conductance. Choosing this parameter appropriately provides a unified approach to generating preferential attachment graphs with and without good expansion processes.

\subsection{The random process}

In [25] we studied a process which generates a sequence of graphs $G_{t}, t=1,2, \ldots, n$. The graph $G_{t}=\left(V_{t}, E_{t}\right)$ has $t$ vertices and $m t$ edges. Here $V_{t}$ is a subset of $t$ random points on $S$, the surface of the sphere in $\boldsymbol{R}^{3}$ of radius $\frac{1}{2 \sqrt{\pi}}$ (so that area $(S)=1$ ). After randomly choosing $x_{t+1} \in S$, it is connected, by preferential attachment (i.e. proportional to degree), to $m$ vertices in $V_{t}$ among those of distance at most $r$ from $x_{t+1}$. We showed that this graph has a power law degree distribution, small seperators and a moderate diameter. In this paper we provide a "smoothed" version of this model. Instead of choosing proportional to degree among those vertices within distance $r$ of $x_{t+1}$, the $m$ neighbors of $x_{t}$ are chosen proportional to degree and some function of the distance to $x_{t+1}$.

Let $F: \mathbf{R}_{+} \rightarrow \mathbf{R}_{+}$. Define

$$
I=\int_{S} F\left(\left|u-u_{0}\right|\right) d u=\frac{1}{2} \int_{x=0}^{\pi} F(x) \sin x d x
$$

where $u_{0}$ is any point in $S$ and $0 \leq\left|u-u_{0}\right| \leq \pi$ is the angular distance from $u$ to $u_{0}$ along a great circle. Other parameters of the process are $m>0$ which denotes the number of edges added in every step and $\alpha \geq 0$ a measure of the bias towards self loops.

- Time step 0: To initialize the process, we start with $G_{0}$ being the Empty Graph.

- Time step $t+1$ : We choose vertex $x_{t+1}$ uniformly at random in $S$ and add it to $G_{t}$. Let

$$
T_{t}\left(x_{t+1}\right)=\sum_{v \in V_{t}} F\left(\left|x_{t+1}-v\right|\right) \operatorname{deg}_{t}(v) .
$$


We add $m$ random edges $\left(x_{t+1}, y_{i}\right), i=1,2, \ldots, m$ incident with $x_{t+1}$. Here, each $y_{i}$ is chosen independently from $V_{t+1}=V_{t} \cup\left\{x_{t+1}\right\}$ (parallel edges and loops are permitted), such that for each $i=1, \ldots, m$, for all $v \in V_{t}$,

$$
\operatorname{Pr}\left(y_{i}=v\right)=\frac{\operatorname{deg}_{t}(v) F\left(\left|x_{t+1}-v\right|\right)}{\max \left(T_{t}\left(x_{t+1}\right), \alpha m I t\right)}
$$

and

$$
\operatorname{Pr}\left(y_{i}=x_{t+1}\right)=1-\frac{T_{t}\left(x_{t+1}\right)}{\max \left(T_{t}\left(x_{t+1}\right), \alpha m I t\right)}
$$

(When $t=0$ we have $\operatorname{Pr}\left(y_{i}=x_{1}\right)=1$.)

For $z>0$ we define

$$
I_{z}=\frac{1}{2} \int_{x=0}^{z} F(x) \sin x d x \text { and } J_{z}=I-I_{z}
$$

We will first prove the following result about the degree distribution and the existence of small separators:

Let $d_{k}(t)$ denote the number of vertices of degree $k$ at time $t$ and let $\bar{d}_{k}(t)$ denote the expectation of $d_{k}(t)$.

\section{Theorem 1}

(a) Suppose that $\alpha>2$ and in addition that

$$
\int_{x=0}^{\pi} F(x)^{2} \sin x d x=O\left(n^{\theta} I^{2}\right)
$$

where $\theta<1$ is a constant.

Then there exists a constant $\gamma_{1}>0$ such that for all $k=k(n) \geq m$,

$$
\bar{d}_{k}(n)=e^{\varphi_{k}(m, \alpha)}\left(\frac{m}{k}\right)^{1+\alpha} n+O\left(n^{1-\gamma_{1}}\right)
$$

where $\varphi_{k}(m, \alpha)=O(1)$ tends to a constant $\varphi_{\infty}(m, \alpha)$ as $k \rightarrow \infty$.

Furthermore, for $n$ sufficiently large, the random variable $d_{k}(n)$ satisfies the following concentration inequality:

$$
\operatorname{Pr}\left(\left|d_{k}(n)-\bar{d}_{k}(n)\right| \geq I^{2} n^{\max \{1 / 2,2 / \alpha\}+\delta}\right) \leq e^{-n^{\delta}} .
$$

(b) Suppose that $\alpha>0$ and $m \geq m_{0}$ where $m_{0}$ is a sufficiently large constant and $\varphi, \eta=o(1)$ are such that $\eta n \rightarrow \infty$ and $J_{\eta} \leq \varphi I$. Then whp, $V_{n}$ can be partitioned into $T, \bar{T}$ such that $|T|,|\bar{T}| \sim n / 2$, and there are $\tilde{O}((\eta+\varphi) m n)$ edges between $T$ and $\bar{T}$.

Remark 1 Note that the exponent in (a) does not depend on the particular function $F$. $F$ manifests itself only through the error terms. 
We now consider the connectivity and diameter of $G_{n}$. For this we will place some more restrictions on $F$.

Define the parameter $\rho(\mu)$ by

$$
I_{\rho}=\mu I
$$

We will say that $F$ is smooth (for some value of $\mu$ ) if

(S1) $F$ is monotone non-increasing.

(S2) $\rho^{2} n \geq L \ln n$ for some sufficiently large constant $L$.

(S3) $\rho^{2} F(2 \rho) \geq c_{3} I$ for some $c_{3}$ which is bounded below.

Property (S3) ensures that if $x_{t}$ is close (within distance $\rho$ ) to an existing vertex $v$, then it has a large enough probability of becoming a neighbor of $v$. This helps to ensure the connectivity of the sub-graph induced by the vertices in $B_{\rho}=\{v \in S:|v-u| \leq \rho\}$. Property (S2) ensures that $B_{\rho}$ contains enough vertices.

Theorem 2 Suppose that $\alpha>2$ and $F$ is smooth for some constant $\mu>0$ and $m \geq$ $K \ln n$ for $K$ sufficiently large. Then whp

(a) $G_{n}$ is connected.

(b) $G_{n}$ has diameter $O(\ln n / \rho)$.

We now consider conditons under which $G_{n}$ is an expander.

Let $F$ be tame if there exist absolute constants $C_{1}, C_{2}$ such that

(T1) $F(x) \geq C_{1}$ for $0<x \leq \pi$.

(T2) $I \leq C_{2}$.

The conductance $\Phi$ of $G_{n}$ is defined by

$$
\Phi=\min _{\operatorname{deg}_{n}(K) \leq m n} \Phi(K)=\min _{\operatorname{deg}_{n}(K) \leq m n} \frac{|E(K: \bar{K})|}{\operatorname{deg}_{n}(K)} .
$$

Theorem 3 If $\alpha>2$ and $F$ is tame and $m \geq K \ln n$ for $K$ sufficiently large then whp

(a) $G_{n}$ has conductance bounded from below by a constant.

(b) $G_{n}$ is connected.

(c) $G_{n}$ has diameter $O\left(\log _{m} n\right)$. 


\subsection{Canonical Functions}

Where possible we will illustrate our theorems using the canonical functions:

$$
\begin{array}{llrl}
F_{0}(u)=1_{|u| \leq r}, & & r \geq n^{\epsilon-1 / 2} . \\
F_{1}(u)=\frac{1}{\max \left\{n^{-\delta}, u\right\}^{\beta}} & & \text { where } \delta<1 / 2 . \\
F_{2}(u)=e^{-\beta u} & & \beta=\beta(n) \geq 0 .
\end{array}
$$

Function $F_{0}$ forces the neighbors of a vertex to be within $r$ of that vertex. Function $F_{1}$ tries to smooth this out in a "polynomial" fashion, discouraging long range attachments. As we will see, there is a qualitative difference between the cases $\beta<2$ and $\beta>2$, the latter behaving more like $F_{0}$ with respect to being an expander. $F_{2}$ tries to discourage long range attachments using an "exponential" smoothing.

Notice also that $F_{0}$ corresponds to the model presented in [25]. Also notice that without the $n^{-\delta}$ term in the definition of $F_{1}$ for $\beta \geq 2$ we would have $I=\infty$. One can justify its inclusion (for some value of $\delta$ ) from the fact that whp the minimum distance between the points in $V_{n}$ is greater than $1 / n \ln n$.

Observe that

$$
\begin{aligned}
& I_{z}\left(F_{0}\right)=\frac{1}{2}(1-\cos (\min \{z, r\})) . \\
& I_{z}\left(F_{1}\right)= \begin{cases}\frac{\beta n^{\delta(\beta-2)}}{4(\beta-2)}+O\left(n^{(\beta-4) \delta}+z^{2-\beta}\right) & z \geq n^{-\delta}, \beta>2 . \\
\Theta\left(z^{2-\beta}\right)+O\left(n^{(\beta-2) \delta}\right) & z \geq n^{-\delta}, \beta<2 \\
\ln \left(n^{\delta} z\right)+O(1) & z \geq n^{-\delta}, \beta=2\end{cases} \\
& I_{z}\left(F_{2}\right)=\frac{1}{2\left(1+\beta^{2}\right)}\left(1-e^{-\beta z}(\cos z+\beta \sin z)\right) .
\end{aligned}
$$

For Part (a) of Theorem 1:

$F=F_{0}: \theta=1-2 \epsilon$.

$F=F_{1}, \beta>2: \theta=2 \delta$.

$F=F_{1}, \beta<2: \theta=0$.

$F=F_{1}, \beta=2: \theta=2 \delta$.

$F=F_{2}: \theta=0$.

For Part (b) of Theorem 1:

$F=F_{0}: \eta=r, \varphi=0$.

$F=F_{1}, \beta>2: \eta=n^{-\delta / 2}, \varphi=O\left(n^{-(\beta-2) \delta / 2}\right)$.

$F=F_{1}, \beta=2: \eta=\frac{\ln \ln n}{\ln n}, \varphi=O(\eta)$. 
As we will see in Theorem $3, F=F_{1}, \beta<2$ does not fit the hypotheses of part (b) of this theorem.

For Theorem 3:

$F=F_{0}: I \sim r^{2} / 4$ and so we can take $\mu \sim 1 / 4, \rho=r / 2, c_{3} \sim 1$.

$F=F_{1}, \beta>2: I \sim \frac{n^{\delta(\beta-2)}}{2(\beta-2)}$ and so we can take $\mu \sim 1 / 4, \rho=n^{-\delta} / 2, c_{3} \sim(\beta-2) / 2$.

$F=F_{1}, \beta<2: I=\Theta(1)$ and we can take $\rho=1, \mu=\Omega(1), c_{3}=\Omega(1)$.

$F=F_{2}: I=\Theta(1)$ and we can take $\rho=1, \mu=\Omega(1), c_{3}=\Omega(1)$.

We have a problem fitting the case of $F_{1}$ with $\beta=2$ into the theorem.

Mihail [32] describes empirical results on the conductance of $G_{n}$ in the case where $F=F_{1}$. They observe poor conductance when $\beta<2$ and good conductance when $\beta>2$. This fits nicely with the results of Theorems 2 and 3 .

We note that $F_{1}$ with $\beta<2$ is tame since $F_{1}(x) \geq \pi^{-\beta}$ for $0 \leq \pi$ and

$$
I=\frac{1}{2} \int_{x=0}^{\pi} \sin x x^{-\beta} d x \leq \frac{\pi^{2-\beta}}{2(2-\beta)} .
$$

\subsection{Open Question, The role of $\alpha$ :}

This parameter was introduced in [25] as a means of overcoming a difficult technical problem. When $\alpha>2$ it facilitates a proof of Lemma 2. On the positive side, it does give a parameter that effects the power law. On the negative side, when $\alpha>2$, there will whp be isolated vertices, unless we make $m$ grow at least as fast as $\ln n$. It is for us, an interesting open question, as to how to prove our results with $\alpha=0$.

\section{Outline of the paper}

We prove a likely power law for the degree sequence in Section 3. We follow a standard practise and prove a recurrence for the expected number of vertices of degree $k$ at time step $t$. Unfortunately, this involves the estimation of the expectation of the reciprocal of a random variable and to handle this, we show that this random variable is concentrated. This is quite technical and is done in Section 3.2. In Section 4 we show that under the assumptions of Theorem 1(b) there are small separators. This is relatively easy, since any give great circle can whp be used to define a small separator.

Section 5 proves connectivity when $m$ grows logarithmically with $n$. The idea is to show that whp the sub-graph $G_{n}(B)$ induced by a ball $B_{\rho}(u)$ of radius $\rho$ centered in $u \in S$, is connected and has small diameter. We then show that the union of the $G_{n}(B)$ 's for $u=x_{1}, x_{2}, \ldots, x_{n}$ is connected and has small diameter.

Section 6 deals with the case of tame functions and shows that whp they give rise to graphs with good expansion properties. 


\section{Proving a power law}

\subsection{Establishing a recurrence for $\bar{d}_{k}(t)$ : the expected number of vertices of degree $k$ at time $t$}

Our approach to proving Theorem 1 (a) is to find a recurrence for $\bar{d}_{k}(t)$. For $k \in \mathbf{N}$ define $D_{k}(t)=\left\{v \in V_{t}: \operatorname{deg}_{t}(v)=k\right\}$. Thus $d_{k}(t)=\left|D_{k}(t)\right|$. Also, define $d_{m-1}(t)=0$ and $\bar{d}_{m-1}(t)=0$ for all integers $t$ with $t>0$. Let $\eta_{k}\left(G_{t}, x_{t+1}\right)$ denote the (conditional) probability that a parallel edge from $x_{t+1}$ to a vertex of degree no more than $k$ is created at time $t+1$. Then,

$$
\eta_{k}\left(G_{t}, x_{t+1}\right)=O\left(\min \left\{\sum_{i=m}^{k} \sum_{v \in D_{i}(t)} \frac{F\left(\left|x_{t+1}-v\right|\right)^{2} i^{2}}{\max \left\{\alpha m I t, T_{t}\left(x_{t+1}\right)\right\}^{2}}, 1\right\}\right) .
$$

We remark that the assumption in equation (1) shows that $\eta_{k}$ is a small quantity, see (11).

Then for $k \geq m$,

$$
\begin{aligned}
& \mathbf{E}\left[d_{k}(t+1) \mid G_{t}, x_{t+1}\right]= d_{k}(t) \\
&+m \sum_{v \in D_{k-1}(t)} \frac{(k-1) F\left(\left|x_{t+1}-v\right|\right)}{\max \left\{\alpha m I t, T_{t}\left(x_{t+1}\right)\right\}}-m \sum_{v \in D_{k}(t)} \frac{k F\left(\left|x_{t+1}-v\right|\right)}{\max \left\{\alpha m I t, T_{t}\left(x_{t+1}\right)\right\}} \\
& \quad+\operatorname{Pr}\left[\operatorname{deg}_{t+1}\left(x_{t+1}=k\right) \mid G_{t}, x_{t+1}\right]+O\left(m \eta_{k}\left(G_{t}, x_{t+1}\right)\right) .
\end{aligned}
$$

Let $\mathcal{A}_{t}$ be the event

$$
\left\{\left|T_{t}\left(x_{t+1}\right)-2 m I t\right| \leq C_{1} I m t^{\gamma} \ln n\right\}
$$

where

$$
\max \{2 / \alpha, \theta\}<\gamma<1
$$

and $C_{1}$ is some sufficiently large constant.

Note that if

$$
t \geq t_{0}=(\ln n)^{2 /(1-\gamma)}
$$

then

$\mathcal{A}_{t}$ implies $T_{t}\left(x_{t+1}\right) \leq \alpha m I t$. 
Then, for $t \geq t_{0}$,

$$
\begin{aligned}
& \mathbf{E}\left[\sum_{v \in D_{k}(t)} \frac{k F\left(\left|x_{t+1}-v\right|\right)}{\max \left\{\alpha m I t, T_{t}\left(x_{t+1}\right)\right\}}\right] \\
& =\mathbf{E}\left[\sum_{v \in D_{k}(t)} \frac{k F\left(\left|x_{t+1}-v\right|\right)}{\max \left\{\alpha m I t, T_{t}\left(x_{t+1}\right)\right\}} \mid \mathcal{A}_{t}\right] \operatorname{Pr}\left[\mathcal{A}_{t}\right] \\
& \quad+\mathbf{E}\left[\sum_{v \in D_{k}(t)} \frac{k F\left(\left|x_{t+1}-v\right|\right)}{\max \left\{\alpha m I t, T_{t}\left(x_{t+1}\right)\right\}} \mid \neg \mathcal{A}_{t}\right] \operatorname{Pr}\left[\neg \mathcal{A}_{t}\right] \\
& =\frac{k}{\alpha m t} \mathbf{E}\left[d_{k}(t) \mid \mathcal{A}_{t}\right] \operatorname{Pr}\left[\mathcal{A}_{t}\right]+O(1) \operatorname{Pr}\left[\neg \mathcal{A}_{t}\right] \\
& =\frac{k \bar{d}_{k}(t)}{\alpha m t}-\frac{k}{\alpha m t} \mathbf{E}\left[d_{k}(t) \mid \neg \mathcal{A}_{t}\right] \operatorname{Pr}\left[\neg \mathcal{A}_{t}\right]+O(1) \operatorname{Pr}\left[\neg \mathcal{A}_{t}\right] \\
& =\frac{k \bar{d}_{k}(t)}{\alpha m t}+O(k) \operatorname{Pr}\left[\neg \mathcal{A}_{t}\right] .
\end{aligned}
$$

In Lemma 2 below we prove that

$$
\operatorname{Pr}\left[\neg \mathcal{A}_{t}\right]=O\left(n^{-2}\right) .
$$

Thus, if $t \geq t_{0}$ then

$$
\mathbf{E}\left[\sum_{v \in D_{k}(t)} \frac{k F\left(\left|x_{t+1}-v\right|\right)}{\max \left\{\alpha m I t, T_{t}\left(x_{t+1}\right)\right\}}\right]=\frac{k \bar{d}_{k}(t)}{\alpha m t}+O\left(k / n^{2}\right) .
$$

In a similar way

$$
\mathbf{E}\left[\sum_{v \in D_{k-1}(t)} \frac{(k-1) F\left(\left|x_{t+1}-v\right|\right)}{\max \left\{\alpha m I t, T_{t}\left(x_{t+1}\right)\right\}}\right]=\frac{(k-1) \bar{d}_{k-1}(t)}{\alpha m t}+O\left(k / n^{2}\right) .
$$

On the other hand, given $G_{t}, x_{t+1}$, if

$$
p=1-\frac{T_{t}\left(x_{t+1}\right)}{\max \left(T_{t}\left(x_{t+1}\right), \alpha m I t\right)}
$$

then

$$
\operatorname{Pr}\left[\operatorname{deg}_{t+1}\left(x_{t+1}\right)=k \mid G_{t}, x_{t+1}\right]=\operatorname{Pr}[\operatorname{Bi}(m, p)=k-m]
$$

So, if $t \geq t_{0}$,

$$
\begin{aligned}
& \operatorname{Pr}\left[\operatorname{deg}_{t+1}\left(x_{t+1}=k\right)\right]=\left(\begin{array}{c}
m \\
k-m
\end{array}\right) \mathbf{E}\left[p^{k-m}(1-p)^{2 m-k} \mid \mathcal{A}_{t}\right] \operatorname{Pr}\left[\mathcal{A}_{t}\right]+O\left(\operatorname{Pr}\left[\neg \mathcal{A}_{t}\right]\right) \\
& =\left(\begin{array}{c}
m \\
k-m
\end{array}\right)\left(1-\frac{2}{\alpha}\right)^{k-m}\left(\frac{2}{\alpha}\right)^{2 m-k}\left(1+O\left(m t^{\gamma-1} \ln n\right)\right) \operatorname{Pr}\left[\mathcal{A}_{t}\right]+O\left(n^{-2}\right) \\
& =\left(\begin{array}{c}
m \\
k-m
\end{array}\right)\left(1-\frac{2}{\alpha}\right)^{k-m}\left(\frac{2}{\alpha}\right)^{2 m-k}+O\left(m t^{\gamma-1} \ln n\right) .
\end{aligned}
$$


Now note that from equations (5) and (8) that if

$$
t \geq t_{1}=n^{(\gamma+\theta) / 2 \gamma}
$$

and

$$
k \leq k_{0}(t)=n^{(\gamma-\theta) / 4}
$$

then, from (1), we see that

$$
\mathbf{E}\left(m \eta_{k}\left(G_{t}, x_{t+1}\right)\right)=O\left(\frac{k^{2} n^{\theta}}{m t}\right)=O\left(t^{\gamma-1}\right) .
$$

Taking expectations on both sides of (6) and using $(9,10,11)$, we see that if $t \geq t_{0}$ and $k \leq k_{0}(t)$ then

$$
\begin{aligned}
\bar{d}_{k}(t+1)=\bar{d}_{k}(t)+\frac{k-1}{\alpha t} & \bar{d}_{k-1}(t)-\frac{k}{\alpha t} \bar{d}_{k}(t) \\
& +\left(\begin{array}{c}
m \\
k-m
\end{array}\right)\left(1-\frac{2}{\alpha}\right)^{k-m}\left(\frac{2}{\alpha}\right)^{2 m-k}+O\left(m t^{\gamma-1} \ln n\right)
\end{aligned}
$$

We consider the recurrence given by $f_{m-1}=0$ and for $k \geq m$,

$$
f_{k}=\frac{k-1}{\alpha} f_{k-1}-\frac{k}{\alpha} f_{k}+\left(\begin{array}{c}
m \\
k-m
\end{array}\right)\left(1-\frac{2}{\alpha}\right)^{k-m}\left(\frac{2}{\alpha}\right)^{2 m-k},
$$

which, for $k>2 m$, has solution

$$
\begin{aligned}
f_{k} & =f_{2 m} \prod_{i=2 m+1}^{k} \frac{i-1}{i+\alpha} \\
& =f_{2 m} e^{\varphi_{k}(m, \alpha)}\left(\frac{m}{k}\right)^{\alpha+1} .
\end{aligned}
$$

Here $\varphi_{k}(m, \alpha)=O(1)$ tends to a limit $\varphi_{\infty}(m, \alpha)$ depending only on $m, \alpha$ as $k \rightarrow \infty$. Furthermore, $\lim _{m \rightarrow \infty} \varphi_{\infty}(\alpha, m)=0$. We also have

$$
f_{m+i}=f_{2 m} \prod_{j=i+1}^{m}\left(1+\frac{\alpha+1}{m+j-1}\right) \leq e^{2 \alpha+3} f_{2 m} .
$$

It follows that (14) is also valid for $m \leq k \leq 2 m$ with $\varphi_{k}(m, \alpha)=O(1)$.

We finish the proof of (2) by showing that there exists a constant $M>0$ such that

$$
\left|\bar{d}_{k}(t)-f_{k} t\right| \leq M\left(t_{1}+m t^{\gamma} \ln n\right)
$$

for all $0 \leq t \leq n$ and $m \leq k \leq k_{0}(t)$.

We have that (15) is trivially true for $t<t_{1}$, and for $t \geq t_{1}$ and $k>k_{0}(t)$ it follows from $\bar{d}_{k}(t) \leq 2 m t / k$.

Now, let $\Psi_{k}(t)=\bar{d}_{k}(t)-f_{k} t$. Then for $t \geq t_{1}$ and $m \leq k \leq k_{0}(t)$,

$$
\Psi_{k}(t+1)=\frac{k-1}{\alpha t} \Psi_{k-1}(t)-\frac{k}{\alpha t} \Psi_{k}(t)+O\left(m t^{\gamma-1} \ln n\right) .
$$


Let $L$ denote the hidden constant in $O\left(m t^{\gamma-1} \ln n\right)$ of (16). Our inductive hypothesis $\mathcal{H}_{t}$ is that

$$
\left|\Psi_{k}(t)\right| \leq M\left(t_{1}+m t^{\gamma} \ln n\right)
$$

for every $m \leq k \leq k_{0}(t)$ and $M$ sufficiently large. Assume that $t \geq t_{1}$. Then $k \ll t$ in the current range of interest, and so from (16),

$$
\begin{aligned}
\left|\Psi_{k}(t+1)\right| & \leq M\left(t_{1}+m t^{\gamma} \ln n\right)+L m t^{\gamma-1} \ln n \\
& \leq M\left(t_{1}+m(t+1)^{\gamma} \ln n\right) .
\end{aligned}
$$

This verifies $\mathcal{H}_{t+1}$ and completes the proof by induction.

\subsection{Concentration of $T_{t}(u)$}

Now we turn our attention to prove that $T_{t}(u)$ is concentrated around its mean.

Lemma 1 Let $u \in S$ and $t>0$ then $\mathbf{E}\left[T_{t}(u)\right]=2 \operatorname{Im} t$

Proof

$$
\mathbf{E}\left[T_{t}(u)\right]=\mathbf{E}\left[\sum_{v \in V_{t}} \operatorname{deg}_{t}(v) F(|u-v|)\right]=I \sum_{v \in V_{t}} \operatorname{deg}_{t}(v)=2 \operatorname{Im} t
$$

Lemma 2 If $t>0$ and $u$ is chosen randomly from $S$ then

$$
\operatorname{Pr}\left[\left|T_{t}(u)-2 \operatorname{Im} t\right| \geq m I\left(t^{2 / \alpha}+t^{1 / 2} \ln t\right) \ln n\right]=O\left(n^{-2}\right) .
$$

Proof We use Azuma-Hoeffding inequality (see for example [2]). One may be a little concerned here that our probability space is not discrete. Although it is not really necessary, one could replace $S$ by $2^{2^{n}}$ randomly chosen points $X$ and sample uniformly from these. Then whp the change in distribution would be negligible. With this reassurance, fix $\tau$, with $1 \leq \tau<t$. Fix $G_{\tau}$ and let $G_{t}=G_{t}\left(G_{\tau}, x_{\tau+1}, y_{1}, \ldots, y_{m}\right)$ and $\hat{G}_{t}=G_{t}\left(G_{\tau}, \hat{x}_{\tau+1}, \hat{y}_{1}, \ldots, \hat{y}_{m}\right)$, where $x_{\tau+1}, \hat{x}_{\tau+1} \in S$ and $y_{1}, \ldots, y_{m}, \hat{y}_{1}, \ldots, \hat{y}_{m} \in V_{\tau}$. We couple the construction of $G_{t}$ and $\hat{G}_{t}$, starting at time step $\tau+1$ with the graph $G_{\tau}$ and $\hat{G}_{\tau}$ respectively. Then, for every step $\sigma>\tau+1$ we choose the same point $x_{\sigma} \in S$ in both and for every $i=1, \ldots, m$ we choose $u_{i}, \hat{u}_{i} \in V_{\sigma}$ such that each marginal is the correct marginal and such that the probability of choosing the same vertex is maximized.

Notice that we have

$$
\operatorname{Pr}\left[u_{i}=v=\hat{u}_{i}\right]=\min \left(\frac{\operatorname{deg}_{G_{\sigma-1}}(v) F\left(\left|v-x_{\sigma}\right|\right)}{\max \left(T_{\sigma-1}\left(x_{\sigma}\right), \alpha m I(\sigma-1)\right)}, \frac{\operatorname{deg}_{\hat{G}_{\sigma-1}}(v) F\left(\left|v-x_{\sigma}\right|\right)}{\max \left(\hat{T}_{\sigma-1}\left(x_{\sigma}\right), \alpha m I(\sigma-1)\right)}\right)
$$

for every $v \in V_{\sigma-1}$. Also,

$$
\operatorname{Pr}\left[u_{i}=x_{\sigma}=\hat{u}_{i}\right]=1-\max \left(\frac{T_{\sigma-1}\left(x_{\sigma}\right)}{\max \left(T_{\sigma-1}\left(x_{\sigma}\right), \alpha m I(\sigma-1)\right)}, \frac{\hat{T}_{\sigma-1}\left(x_{\sigma}\right)}{\max \left(\hat{T}_{\sigma-1}\left(x_{\sigma}\right), \alpha m I(\sigma-1)\right)}\right)
$$


Now, for $u \in S$ let

$$
\Delta_{\sigma}(u):=\Delta_{\sigma, \tau}(u)=\sum_{\rho=\tau}^{\sigma} \sum_{i=1}^{m}\left|F\left(\left|u-u_{i}^{\rho}\right|\right)-F\left(\left|u-\hat{u}_{i}^{\rho}\right|\right)\right| .
$$

Lemma 3 Let $t \geq 1$ and let $u$ be a random point in $S$. Then for some constant $C>0$,

$$
\mathbf{E}\left[\Delta_{t}(u)\right] \leq C m I\left(\frac{t}{\tau}\right)^{2 / \alpha}
$$

Proof We begin with

$$
\mathbf{E}\left[\left|F\left(\left|w-u_{i}^{\rho}\right|\right)-F\left(\left|w-\hat{u}_{i}^{\rho}\right|\right)\right| \mid u_{i}^{j}, \hat{u}_{i}^{j}: i=1, \ldots, m, j=1, \ldots, \sigma\right] \leq 2 I 1_{u_{i}^{\rho} \neq \hat{u}_{i}^{\rho}} .
$$

Therefore if we define for every $\tau<\sigma \leq t$

$$
\Delta_{\sigma}=\sum_{\rho=\tau}^{\sigma} \sum_{i=1}^{m} 1_{u_{i}^{\sigma} \neq \hat{u}_{i}^{\sigma}},
$$

we have

$$
\mathbf{E}\left[\Delta_{\sigma}(u)\right] \leq 2 I \mathbf{E}\left[\Delta_{\sigma}\right]
$$

Fix $\tau<\sigma \leq t$. We have then

$$
\Delta_{\sigma}=\Delta_{\sigma-1}+\sum_{i=1}^{m} 1_{u_{i}^{\sigma} \neq \hat{u}_{i}^{\sigma}} .
$$

Now fix $1 \leq i \leq m$. Taking expectations with respect to our coupling,

$$
\begin{aligned}
\mathbf{E} & {\left[1_{u_{i}^{\sigma} \neq \hat{u}_{i}^{\sigma}} \mid G_{\sigma-1}, \hat{G}_{\sigma-1}, x_{\sigma}\right]=1-\mathbf{P r}\left[u_{i}^{\sigma}=\hat{u}_{i}^{\sigma} \mid G_{\sigma-1}, \hat{G}_{\sigma-1}, x_{\sigma}\right] } \\
= & \max \left(\frac{T_{\sigma-1}\left(x_{\sigma}\right)}{\max \left(T_{\sigma-1}\left(x_{\sigma}\right), \alpha m I(\sigma-1)\right)}, \frac{\hat{T}_{\sigma-1}\left(x_{\sigma}\right)}{\max \left(\hat{T}_{\sigma-1}\left(x_{\sigma}\right), \alpha m I(\sigma-1)\right)}\right) \\
& -\sum_{v \in V_{\sigma-1}} \min \left(\frac{\operatorname{deg}_{G_{\sigma-1}}(v) F\left(\left|v-x_{\sigma}\right|\right)}{\max \left(T_{\sigma-1}\left(x_{\sigma}\right), \alpha m I(\sigma-1)\right)}, \frac{\operatorname{deg}_{\hat{G}_{\sigma-1}}(v) F\left(\left|v-x_{\sigma}\right|\right)}{\max \left(\hat{T}_{\sigma-1}\left(x_{\sigma}\right), \alpha m I(\sigma-1)\right)}\right) \\
\leq & \frac{\max \left(T_{\sigma-1}\left(x_{\sigma}\right), \hat{T}_{\sigma-1}\left(x_{\sigma}\right)\right)-\sum_{v \in V_{\sigma-1}} \min \left(\operatorname{deg}_{G_{\sigma-1}}(v), \operatorname{deg}_{\hat{G}_{\sigma-1}}(v)\right) F\left(\left|v-x_{\sigma}\right|\right)}{\max \left(T_{\sigma-1}\left(x_{\sigma}\right), \hat{T}_{\sigma-1}\left(x_{\sigma}\right), \alpha m I(\sigma-1)\right)} \\
\leq & \frac{\sum_{v \in V_{\sigma-1}}\left|\operatorname{deg}_{G_{\sigma-1}}(v)-\operatorname{deg}_{\hat{G}_{\sigma-1}}(v)\right| F\left(\left|v-x_{\sigma}\right|\right)}{\max \left(T_{\sigma-1}\left(x_{\sigma}\right), \hat{T}_{\sigma-1}\left(x_{\sigma}\right), \alpha m I(\sigma-1)\right)} \\
\leq & \frac{\sum_{v \in V_{\sigma-1}}\left|\operatorname{deg}_{G_{\sigma-1}}(v)-\operatorname{deg}_{\hat{G}_{\sigma-1}}(v)\right| F\left(\left|v-x_{\sigma}\right|\right)}{\alpha m I(\sigma-1)}
\end{aligned}
$$


Inequality (18), follows from

$$
\max \left(\frac{a}{\max (a, c)}, \frac{b}{\max (b, c)}\right)=\frac{\max (a, b)}{\max (a, b, c)}
$$

and

$$
\min \left(\frac{a}{b}, \frac{c}{d}\right) \geq \frac{\min (a, c)}{\max (b, d)} .
$$

Inequality (19) is a consequence of $\max \left\{\sum_{i} a_{i}, \sum_{i} b_{i}\right\}-\sum_{i} \min \left\{a_{i}, b_{i}\right\} \leq \sum_{i}\left|a_{i}-b_{i}\right|$.

Therefore

$$
\mathbf{E}\left[\Delta_{\sigma} \mid G_{\sigma-1}, \hat{G}_{\sigma-1}\right] \leq \Delta_{\sigma-1}+\frac{\sum_{v \in V_{\sigma-1}}\left|\operatorname{deg}_{G_{\sigma-1}}(v)-\operatorname{deg}_{\hat{G}_{\sigma-1}}(v)\right|}{\alpha(\sigma-1)} .
$$

But, for each $v \in V_{\sigma-1}$ we have

$$
\left|\operatorname{deg}_{G_{\sigma-1}}(v)-\operatorname{deg}_{\hat{G}_{\sigma-1}}(v)\right| \leq \sum_{j=\tau}^{\sigma-1} \sum_{i=1}^{m}\left(1_{u_{i}^{j}=v, \hat{u}_{i}^{j} \neq v}+1_{u_{i}^{j} \neq v, \hat{u}_{i}^{j}=v}\right)
$$

and thus

$$
\sum_{v \in V_{\sigma-1}}\left|\operatorname{deg}_{G_{\sigma-1}}(v)-\operatorname{deg}_{\hat{G}_{\sigma-1}}(v)\right| \leq \sum_{j=\tau}^{\sigma-1} \sum_{i=1}^{m} \sum_{v \in V_{\sigma-1}}\left(1_{u_{i}^{j}=v, \hat{u}_{i}^{j} \neq v}+1_{u_{i}^{j} \neq v, \hat{u}_{i}^{j}=v}\right) \leq 2 \Delta_{\sigma-1} .
$$

Going back to (20) we have

$$
\mathbf{E}\left[\Delta_{\sigma}\right] \leq \mathbf{E}\left[\Delta_{\sigma-1}\right]\left(1+\frac{2}{\alpha(\sigma-1)}\right),
$$

so, $\mathbf{E}\left[\Delta_{t}\right] \leq e^{O(1)}\left(\frac{t}{\tau}\right)^{2 / \alpha} \mathbf{E}\left[\Delta_{\tau}\right]$. Now, $\Delta_{\tau} \leq m$, because the graphs $G_{\tau}$ and $\hat{G}_{\tau}$ differ at most in the last $m$ edges. Therefore $\mathbf{E}\left[\Delta_{t}\right] \leq e^{O(1)} m\left(\frac{t}{\tau}\right)^{2 / \alpha}$.

To apply Azuma's inequality we note first that

$$
\left|\mathbf{E}_{G_{t}}\left[T_{t}(u)\right]-\mathbf{E}_{\hat{G}_{t}}\left[T_{t}(u)\right]\right|=\left|\mathbf{E}\left[\sum_{\rho=\tau}^{t} \sum_{i=1}^{m}\left(F\left(\left|u-u_{i}^{\rho}\right|\right)-F\left(\left|u-\hat{u}_{i}^{\rho}\right|\right)\right)\right]\right| \leq \mathbf{E}\left[\Delta_{t}(u)\right],
$$

and from Lemma 3

$$
\sum_{\tau=1}^{t} \mathbf{E}\left[\Delta_{t}(u)\right]^{2} \leq\left(e^{O(1)} m I\right)^{2} t^{4 / \alpha} \sum_{\tau=1}^{t} \tau^{-4 / \alpha}=O\left(I^{2} m^{2}\left(t \ln t+t^{4 / \alpha}\right)\right)
$$

Therefore, there is $C_{1}$ such that

$$
\operatorname{Pr}\left[\left|T_{t}(u)-\mathbf{E}\left[T_{t}(u)\right]\right| \geq C_{1} \operatorname{Im}\left(t^{2 / \alpha}+t^{1 / 2} \ln t\right)(\ln n)^{1 / 2}\right] \leq e^{-2 \ln n}=n^{-2} \cdot \square
$$




\subsection{Concentration of $d_{k}(t)$}

We follow the proof of Lemma 3, replacing $T_{t}(u)$ by $d_{k}(t)$ and using the same coupling, When we reach (21) we find that $\left|\mathbf{E}_{G_{t}}\left[d_{k}(t)\right]-\mathbf{E}_{\hat{G}_{t}}\left[d_{k}(t)\right]\right| \leq 2 \mathbf{E}\left[\Delta_{t}\right]$, the rest is the same.

This proves (1) and completes the proof of Theorem 1(a) .

\section{Small separators}

In this section we prove Theorem 1(b). For this, we assume $\alpha>0$ and $m_{0} \leq m$ where $m_{0}$ is a sufficiently large constant and $\varphi, \eta=o(1)$ are such that $\eta n \rightarrow \infty$ and $J_{\eta} \leq \varphi I$.

We use the geometry of the instance to obtain a sparse cut. Consider partitioning the vertices in $V_{n}$ using a great circle of $S$. This will divide $V_{n}$ into sets $T$ and $\bar{T}$ which each contain about $n / 2$ vertices. More precisely, we have

$$
\operatorname{Pr}[|T|<(1-\xi) n / 2]=\operatorname{Pr}[|\bar{T}|<(1-\xi) n / 2] \leq e^{-\xi^{2} n / 4} .
$$

To bound $e(T, \bar{T})$, the number of edges crossing the cut, we divide the edges into two types. We call an edge $\{u, v\}$ in $G_{n}$ long if $|u-v| \geq \eta$, otherwise we call it short. We will show that whp the number of long edges is small, and therefore we just need to consider short edges in a cut. Let $Z$ denote the number of long edges. Then

$$
\begin{aligned}
\mathbf{E}[Z] & \leq m t_{0}+m \sum_{t \geq t_{0}} \sum_{v \in V_{t}} \frac{\operatorname{deg}_{t}(v) J_{\eta}}{\alpha m I t} \\
& \leq m t_{0}+m \sum_{t \geq t_{0}} \frac{J_{\eta}}{\alpha I} \\
& =m t_{0}+O(m n \varphi) .
\end{aligned}
$$

Now whp there are at most $\mathbf{E}[Z] / \varphi^{1 / 2}$ long edges. Apart from these, edges only appear between vertices within distance $\eta$, so only edges incident with vertices appearing in the strip within distance $\eta$ of the great circle can appear in the cut. Since $\eta=o(1)$, this strip has area less than $3 \eta \sqrt{\pi}$, and, letting $U$ denote the vertices appearing in this strip, we have

$$
\operatorname{Pr}[|U| \geq 4 \sqrt{\pi} \eta n] \leq e^{-\sqrt{\pi} \eta n / 9}=o(1) .
$$

Even if every one of the vertices chooses its $m$ neighbors on the opposite side of the cut, this will yield at most $4 \sqrt{\pi} \eta n m$ edges whp. So the graph has a cut with

$$
e(T, \bar{T})=\tilde{O}\left(\left(\eta+\varphi^{1 / 2}\right) m n\right)
$$

with probability at least $1-o(1)$.

\section{Connectivity and Diameter}

Here we prove Theorem 2. Let $\mu$ be such that $F$ is smooth for $\mu$, and let $\rho=\rho(\mu)$. Fix $u \in S$ let

$$
B_{\rho}=\{v \in S:|v-u| \leq \rho\}
$$


and let $A_{\rho}=\int_{v \in B_{\rho}} d v \in\left[c_{1} \rho^{2}, c_{2} \rho^{2}\right]$ denote the area of $B_{r}$. Here $c_{1}, c_{2}$ are some absolute constants, independent of $\rho$.

We denote the diameter of $G$ by $\operatorname{diam}(G)$, and follow the convention of defining $\operatorname{diam}(G)=\infty$, when $G$ is disconnected. In particular, when we say that a graph has finite diameter this implies it is connected.

Let

$$
T=\frac{K_{1} \ln n}{A_{\rho}} \leq \frac{K_{1} n}{c_{1} L}
$$

where $L$ is as in $\mathbf{S} 2$ and $K_{1}$ is sufficiently large, and $L^{2 / 3} \ll K_{1} \ll K, L$.

\section{Lemma 4}

$$
\operatorname{Pr}\left[\operatorname{diam}\left(G_{n}\left(B_{\rho}\right)\right) \geq 2\left(K_{1}+1\right) \ln n\right]=O\left(n^{-1}\right)
$$

where $G_{n}\left(B_{\rho}\right)$ is the induced subgraph of $G_{n}$ in $B_{\rho}$.

Proof Let $N=\left|G_{n}\left(B_{\rho}\right)\right|$ and let $V\left(G_{n}\left(B_{\rho}\right)\right)=\left\{x_{t_{1}}, \ldots, x_{t_{N}}\right\}$, where $t_{s}<t_{s+1}$ for all $s<N$ and $t_{N} \leq n$. For $s=1, \ldots, N$ let $H_{s}=G_{t_{s}}\left(B_{\rho}\right)$. We concentrate our attention to the evolution of $H_{s}$.

Notice that $s$ is the number of steps for which $x_{t} \in B_{\rho}$ with $t \leq t_{s}$, and so $s \sim$ $\operatorname{Bi}\left(t_{s}, A_{\rho}\right)$. By the Chernoff bound we have that if $t_{s} \geq T$,

$$
\operatorname{Pr}\left[\frac{1}{2}<\frac{t_{s} A_{\rho}}{s}<\frac{3}{2}\right] \geq 1-n^{-K_{1} / 13} .
$$

Therefore, if $N_{0}$ is the number of vertices in $B_{\rho}$ at time $T$, we may assume for all $s \geq N_{0}$, $s / 2<t_{s} A_{\rho}<3 s / 2$. In particular, $N \geq 2 n A_{\rho} / 3 \geq c_{1} L \ln n / 2$ and $N_{0} \leq 2 T A_{\rho} \leq 2 K_{1} \ln n$.

Let $X_{s}$ be the number of connected components of $H_{s}$. Then

$$
X_{s+1}=X_{s}-Y_{s}+1, \quad X_{0}=0
$$

where $Y_{s} \geq 0$ is the number of components conected to $x_{t_{s}}$.

$B_{\rho}$ is contained in $B_{2 \rho}\left(x_{t_{s}}\right)$ the ball of radius $2 \rho$ centered at $x_{t_{s}}$. Therefore if $v \in$ $B_{\rho} \cap V_{t_{s}}$ and $t_{s}>T$,

$\operatorname{Pr}\left[x_{t_{s}}\right.$ chooses $\left.v\right] \geq \frac{\operatorname{deg}_{t_{s}}(v) F\left(\left|x_{t_{s}}-v\right|\right)}{\alpha m I t_{s}} \geq \frac{F(2 \rho)}{\alpha I t_{s}} \geq \frac{2 A_{\rho} F(2 \rho)}{3 \alpha I s} \geq \frac{2 c_{1} \rho^{2} F(2 \rho)}{3 \alpha I s} \geq \frac{2 c_{1} c_{3}}{3 \alpha s}$.

The last inequality uses S3.

Now, we can bound the probability of generating a new component,

$$
\begin{aligned}
\operatorname{Pr}\left[Y_{s}=0 \mid H_{s-1}\right]= & \left(1-\sum_{v \in H_{s-1}} \operatorname{Pr}\left[x_{t_{s}} \text { chooses } v\right]\right)^{m} \\
& \leq\left(1-\frac{2 c_{1} c_{3}}{3 \alpha}\right)^{m} \leq \exp \left(-\frac{2 c_{1} c_{3} m}{3 \alpha}\right) \leq n^{-10}
\end{aligned}
$$


If $s<2 K_{1} \ln n$, as $m \geq K \ln n$, we can bound the probability of not collapsing components,

$$
\begin{aligned}
\operatorname{Pr}\left[Y_{s}=1 \mid X_{s} \geq 2\right] & \leq \operatorname{Pr}\left[Y_{s}=1 \mid X_{s} \geq 2, Y_{s}>0\right]+\operatorname{Pr}\left[Y_{s}=0 \mid X_{s} \geq 2\right] \\
& \leq 2\left(1-\frac{2 c_{1} c_{3}}{3 \alpha s}\right)^{m}+n^{-10} \\
& \leq 2 \exp \left(-\frac{2 m c_{1} c_{3}}{3 \alpha s}\right)+n^{-10} \leq 1 / 10
\end{aligned}
$$

Therefore, $X_{s}$ is stochastically dominated by the random variable $\max \left\{1, N_{0}-Z_{s}\right\}$ where $Z_{s} \sim \operatorname{Bi}(s, 9 / 10)$. We then have

$$
\operatorname{Pr}\left[X_{4 K_{1} \ln n}>1\right] \leq \operatorname{Pr}\left[Z_{4 K_{1} \ln n}<N_{0}\right] \leq \operatorname{Pr}\left[Z_{4 K_{1} \ln n}<2 K_{1} \ln n\right] \leq n^{-3} .
$$

And therefore

$$
\operatorname{Pr}\left[H_{4 K_{1} \ln n} \text { is not connected }\right] \leq n^{-3} .
$$

Now, to obtain an upper bound on the diameter, we run the process of construction of $H_{N}$ by rounds. The first round consists of $4 K_{1} \ln n$ steps and in each new round we double the size of the graph, i.e. it consists of as many steps as the total number of steps of all the previous rounds. Notice that we have less than $\log _{2} n$ rounds in total. Let $\mathcal{A}$ be the event for all $i>0$ every vertex created in the $(i+1)$ th round is adjacent to a vertex in $H_{2^{i+1} K_{1} \ln n}$, the graph at the end of the $i$ th round.

On the event $\mathcal{A}$, every vertex in $H_{N}$ is at distance at $\operatorname{most} \log _{2} n$ of $H_{2 K_{1} \ln n}$ whose diameter is not greater than $2 K_{1} \ln n$. Thus, the diameter of $H_{N}$ is smaller than $2\left(K_{1}+\right.$ 2) $\ln n$.

Now, if $v$ is created in the $(i+1)$ st round,

$$
\operatorname{Pr}\left[v \text { is not adjacent to } H_{2^{i-1} K_{1} \ln n}\right] \leq\left(1-\frac{2 c_{1} c_{3}}{3 \alpha}\right)^{m} .
$$

Therefore

$$
\operatorname{Pr}[\neg \mathcal{A}] \leq\left(1-\frac{2 c_{1} c_{3}}{3 \alpha}\right)^{m} n(\ln n) \leq n^{1+o(1)-2 K c_{1} c_{3} /(3 \alpha)}
$$

To finish the proof of connectivity and the diameter, let $u, v$ be two vertices of $G_{n}$. Let $C_{1}, C_{2}, \ldots, C_{M}, M=O(1 / \rho)$ be a sequence of spherical caps of radius $\rho$ such that $u$ is the center of $C_{1}, v$ is the center of $C_{M}$ and such that the centers of $C_{i}, C_{i+1}$ are distance $\leq \rho / 2$ apart. The intersections of $C_{i}, C_{i+1}$ have area at least $A_{\rho} / 10$ and so whp each intersection contains a vertex. Using Lemma 4 we deduce that whp there is a path from $u$ to $v$ in $G_{n}$ of size at most $O(\ln n / \rho)$.

\section{Proof of Theorem 3}

For a set $K \subseteq V_{n}$ we define $\operatorname{deg}_{n}(K)=\sum_{v \in K} \operatorname{deg}_{n}(v)$. 
Lemma 5 There is an absolute constant $0<\xi<1 / 4$ such that

$$
\operatorname{Pr}\left(\exists K \subseteq V_{n},|K| \geq(1-\xi) n: \operatorname{deg}_{n}(K) \leq(1+\xi) m n\right)=o\left(n^{-3}\right) .
$$

Proof Let $\zeta$ be a small positive constant and divide $V_{n}$ into approximately $1 / \zeta$ sets $S_{1}, S_{2}, \ldots$ of size $s=\lceil\zeta n\rceil$ plus a set of $n-\lfloor 1 / \zeta\rfloor s$ where $S_{i}=\left\{x_{(i-1) s+1}, \ldots, x_{i s}\right\}$, $i=1,2, \ldots$, . We put a high probability upper bound on $\operatorname{deg}_{n}\left(S_{1}\right)$. Now consider the random variables $\beta_{k}, k=2, \ldots$ where $\beta_{k}=\operatorname{deg}_{\tau_{k}}\left(S_{2} \cup \cdots S_{k}\right) / m s$ and $\tau_{k}=k s$. Now $\beta_{2} \geq m s$ and conditional on the value of $\beta_{k} \geq(k-1) m s$

$$
\beta_{k+1} m s \text { dominates } m s+\beta_{k} m s+\operatorname{Bi}\left(m s, \frac{\beta_{k} \lambda}{2(k+1)}\right)
$$

where $\lambda=C_{1} / C_{2} . C_{1}, C_{2}$ are in the definition of tameness.

So, there exist constants $\gamma_{1}, \gamma_{2}$ (independent of $\zeta$ ) such that

$$
\operatorname{Pr}\left(\frac{\beta_{k+1}}{m s} \leq 1+\left(1+\gamma_{2}\right) \frac{\beta_{k}}{m s}\right) \leq e^{-m \gamma_{1} n} .
$$

So, after some calculations, we find that with probability $1-O\left(e^{-m \gamma_{1} n}\right)$,

$$
\operatorname{deg}_{n}\left(V_{n} \backslash S_{1}\right) \geq m s\left(1+\gamma_{2}\right) \gamma_{2}^{-1}\left(\left(1+\gamma_{2}\right)^{\lfloor 1 / \zeta\rfloor-3}-1\right) \geq m n(1+\zeta / 2)
$$

for small enough $\zeta$.

Now $\operatorname{deg}_{n}\left(S_{1}\right) \operatorname{dominates} \operatorname{deg}_{n}(L)$ for any set $L$ of size $\lceil\zeta n\rceil$. So, if $m>1 / \gamma_{1}$ then the probability there is a set of size $\lceil\zeta n\rceil$ which has total degree exceeding $m n(1-$

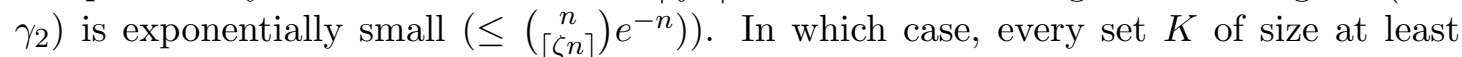
$n-\lceil\zeta n\rceil$ has total degree $\operatorname{deg}_{n}(K) \geq m n\left(1+\gamma_{2} / 2\right)$ and the lemma follows by taking $\xi=\min \left\{\zeta, \gamma_{2} / 2,1 / 4\right\}$.

We have to estimate $\Phi(K)$ for all $K$ with $\operatorname{deg}_{n}(K) \leq m n$. The above lemma shows that we can restrict our attention to sets $K$ with $|K| \leq(1-\xi) n$.

We now observe that for $K \subseteq V_{n}$,

$$
\operatorname{deg}_{n}(K)=m|K|+|E(K: \bar{K})|
$$

and so to bound $\Phi(K)$, it suffices to prove lower bounds $|E(K: \bar{K})| \geq \eta m|K|$ for some positive constant $\eta$.

Lemma 6 If $m \geq C \ln n$ where $C$ is sufficiently large then there exists an absolute constant $\kappa>0$ such that

$$
\operatorname{Pr}\left(\Phi\left(G_{n}\right)<\kappa\right)=O\left(n^{-3}\right) .
$$

\section{Proof}

Case 1: $1 \leq|K| \leq A_{0} n$.

Here $A_{0}$ is a sufficiently small constant. Let $K_{1}=K \cap V_{n / 2}$ and $K_{2}=K \backslash K_{1}$. Let $W_{1}=V_{n / 2} \backslash K_{1}$ and $W_{2}=V_{n} \backslash\left(V_{n / 2} \cup K_{2}\right)$. The number of edges between $K_{1}$ and $W_{2}$ dominates $\operatorname{Bi}\left(m\left(n / 2-\left|K_{2}\right|\right), \lambda\left|K_{1}\right| /(\alpha n)\right)$. This is because each edge chosen 
by $V_{j}, j \in W_{2}$ has probability at least $m \lambda\left|K_{1}\right| /(\alpha m n)$ of being in $K_{1}$. Similarly, the number of edges between $K_{2}$ and $W_{1}$ dominates $\operatorname{Bi}\left(m\left|K_{2}\right|, \lambda\left(n / 2-\left|K_{1}\right|\right) /(\alpha n)\right)$. Thus $\mathbf{E}[|E(K: \bar{K})|] \geq m \lambda|K| /(3 \alpha)$ and so by Hoeffdings inequality we see that $|E(K: \bar{K})| \geq$ $m \lambda|K| /(4 \alpha)$ with probability $1-e^{-c m \lambda|K|}$ for some constant $c=c(\alpha)$. Thus

$$
\operatorname{Pr}\left(\exists K, 1 \leq|K| \leq A_{0} n,|E(K: \bar{K})|<m \lambda|K| /(4 \alpha)\right) \leq \sum_{k=1}^{A_{0} n}\left(\begin{array}{l}
n \\
k
\end{array}\right) e^{-c C \lambda k \ln n}=o(1)
$$

if $C \geq 2 /(c \lambda)$.

Case 2: $A_{0} n \leq|K| \leq(1-\xi) n$.

Here $\xi$ is as in Lemma 5. Let $K_{1}, K_{2}, W_{1}, W_{2}$ be as in Case 1 . Let $q=\left|K_{1}\right|$ and $r=$ $\left|K_{2}\right|$. We calculate the expected number of edges $\mu\left(K_{1}, K_{2}\right)$ of $L=\left(K_{2} \times W_{1} \cup W_{2} \times K_{1}\right)$ generated at steps $\tau, n / 2 \leq \tau \leq n$ which are directed into $K$. At step $\tau$ the number of such edges falling in $L$ is an independent random variable with distribution dominating

$$
1_{\tau \in W_{2}} \operatorname{Bi}\left(m, \frac{\lambda q}{\alpha \tau}\right)+1_{\tau \in K_{2}} \operatorname{Bi}\left(m, \frac{\lambda(n / 2-q)}{\alpha \tau}\right) .
$$

Thus

$$
\begin{aligned}
\mu\left(K_{1}, K_{2}\right) & \geq \frac{m \lambda q}{\alpha} \sum_{\tau \in W_{2}} \frac{1}{\tau}+\frac{m \lambda(n / 2-q)}{\alpha} \sum_{\tau \in K_{2}} \frac{1}{\tau} \\
& =\frac{m \lambda}{\alpha}\left((k-r) \sum_{\tau \in W_{2}} \frac{1}{\tau}+(n / 2-(k-r)) \sum_{\tau \in K_{2}} \frac{1}{\tau}\right) .
\end{aligned}
$$

Let $\mu(k)=\min _{K_{1}, K_{2}} \mu\left(K_{1}, K_{2}\right)$. Then 'somewhat crudely'

$$
\begin{aligned}
\sum_{\tau \in W_{2}} \frac{1}{\tau} \geq \ln \frac{n}{n / 2+r} \\
\sum_{\tau \in K_{2}} \frac{1}{\tau} \geq \ln \frac{n}{n-r}
\end{aligned}
$$

Thus

$$
\mu(k) \geq \frac{m \lambda}{\alpha}\left((k-r) \ln \frac{2 n}{n+2 r}+\left(\frac{n}{2}-(k-r)\right) \ln \frac{n}{n-r}\right) .
$$

Putting $k=\kappa n$ and $r=\rho n$ we see that

$$
\mu(k) \geq \frac{\lambda m n}{\alpha} g(\kappa, \rho)
$$

where

$$
g(\kappa, \rho)=(\kappa-\rho) \ln \frac{2}{1+2 \rho}+\left(\frac{1}{2}-\kappa+\rho\right) \ln \frac{1}{1-\rho} .
$$

We put a lower bound on $g$ :

$$
\rho \leq \frac{\xi}{2} \text { implies } \kappa-\rho \geq \frac{\xi}{2} \text { and so } g(\kappa, \rho) \geq \frac{\xi}{2} \ln \frac{2}{1+\xi} .
$$


So we can assume that $\rho \geq \xi / 2$. Then

$$
\begin{array}{rlll}
\kappa-\rho \leq \frac{1-\xi}{2} & \text { implies } & g(\kappa, \rho) \geq \frac{\xi}{2} \ln \frac{2}{2-\xi} . \\
\kappa-\rho>\frac{1-\xi}{2} \text { and } \rho \leq \frac{1-\xi}{2} & \text { implies } & g(\kappa, \rho) \geq \frac{1-\xi}{2} \ln \frac{2}{2-\xi} . \\
\kappa-\rho>\frac{1-\xi}{2} \text { and } \rho>\frac{1-\xi}{2} & \text { implies } & \kappa>1-\xi .
\end{array}
$$

We deduce that within our range of interest,

$$
\mu(k) \geq \eta m n
$$

for some absolute constant $\eta$.

Let $Z$ be the number of edges generated within $L$, so that $Z$ counts a subset of the edges between $K$ and $\bar{K}$. Then

$$
\operatorname{Pr}\left(\exists K_{1}, K_{2} \subseteq N: Z \leq \frac{1}{2} \eta m n\right) \leq 2^{n} e^{-\eta m n / 8} \leq e^{-\eta m t / 10}=o(1) .
$$

This completes the proof of Theorem 3(a). Part (b) is an immediate consequence of Part (a).

To prove part (c) we need to prove some vertex expansion properties of $G_{n}$. So fix $K \subseteq V_{n}$ with $1 \leq|K| \leq A_{0} n$ and go back to Case 1 . We see that the number of neighbors of $K_{1}$ in $W_{2}$ dominates $B_{1}=\operatorname{Bi}\left(n / 2-\left|K_{2}\right|, 1-\left(1-\lambda\left|K_{1}\right| /(\alpha n)\right)^{m}\right)$ and the number of neighbours of $K_{2}$ in $W_{1}$ dominates $B_{2}=\operatorname{Bi}\left(n / 2-\left|K_{1}\right|, 1-(1-\lambda /(\alpha n))^{m\left|K_{2}\right|}\right)$. So, for $i=1,2$,

$$
\mathbf{E}\left[B_{i}\right] \geq \begin{cases}\frac{\lambda m\left|K_{i}\right|}{3 \alpha} & \text { if } \frac{\lambda m\left|K_{i}\right|}{\alpha n} \leq \frac{1}{10} \\ \frac{n}{60} & \text { otherwise }\end{cases}
$$

Therefore, using the Chernoff bounds, we have

$$
\begin{aligned}
\operatorname{Pr}\left(\exists K, i: 1 \leq\left|K_{i}\right| \leq \frac{\alpha n}{10 \lambda m} \text { and } B_{i} \leq \frac{\lambda m\left|K_{i}\right|}{6 \alpha}\right) & \leq \sum_{k=1}^{\alpha n /(10 \lambda m)}\left(\begin{array}{l}
n \\
k
\end{array}\right) e^{-\lambda m k /(24 \alpha)} \\
& =o(1) \\
\operatorname{Pr}\left(\exists K, i: \frac{\alpha n}{10 \lambda m} \leq\left|K_{i}\right| \leq A_{0} n \text { and } B_{i} \leq \frac{n}{120}\right) & \leq \sum_{k=1}^{A_{0} n}\left(\begin{array}{l}
n \\
k
\end{array}\right) e^{-n / 1000} \\
& =o(1) .
\end{aligned}
$$

Now fix $x, y \in V_{n}$ and then for $a=x, y$ let $S_{i, a}=\left\{z \in V_{n}: \operatorname{dist}(a, z)=i\right\}$. Here $\operatorname{dist}(a, z)$ is the graph distance between $a$ and $z$ in $G_{n}$. It follows from (22) and (23) that there exists $j_{a}=O\left(\log _{m} n\right)$ such that $\left|S_{j, a}\right| \geq n / 120$. It follows from the proof of Lemma 6 that if $\left|S_{j_{a}}\right| \leq(1-\xi) n$ then $\left|E\left(S_{j_{a}}: \bar{S}_{j_{a}}\right)\right| \geq \eta m n / 120$. It follows that there exists $l_{a} \leq 240 / \eta$ such that $\left|S_{j_{a}+l_{a}}\right| \geq(1-\xi) n \geq 3 n / 4$. It follows that $S_{j_{x}+l_{x}} \cap S_{j_{y}+l_{y}} \neq \emptyset$ and $\operatorname{dist}(x, y) \leq j_{x}+j_{y}+l_{x}+l_{y}=O\left(\log _{m} n\right)$. This completes the proof of Theorem 3 .

Acknowledgement: We thank Henri van den Esker for pointing out some errors and for some very useful comments. We also thank the referees for their comments. 


\section{References}

[1] W. Aiello, F. R. K. Chung, and L. Lu, A random graph model for massive graphs, Proc. of the 32nd Annual ACM Symposium on the Theory of Computing, (2000) 171-180.

[2] N. Alon and J. Spencer, The Probabilistic Method, Second Edition, WileyInterscience 2000 .

[3] W. Aiello, F. R. K. Chung, and L. Lu, Random Evolution in Massive Graphs, Proc. of IEEE Symposium on Foundations of Computer Science, (2001) 510-519.

[4] R. Albert, A. Barabási, and H. Jeong, Diameter of the world wide web, Nature 401 (1999) 103-131.

[5] A. Barabasi and R. Albert, Emergence of scaling in random networks, Science 286 (1999) 509-512.

[6] N. Berger, B. Bollobas, C. Borgs, J. Chayes, and O. Riordan, Degree distribution of the FKP network model, Proc. of the 30th International Colloquium of Automata, Languages and Programming, (2003) 725-738.

[7] N. Berger, C. Borgs, J. Chayes, R. D’Souza, and R. D. Kleinberg, Competitioninduced preferential attachment, Proceedings of the 31st International Colloquium on Automata, Languages and Programming (ICALP), 208-221, Lecture Notes in Computer Science 3142 (2004).

[8] D. Blandford, G. E. Blelloch, and I. Kash, Compact Representations of Separable Graphs, Proc. of ACM/SIAM Symposium on Discrete Algorithms (2003) 679-688.

[9] B. Bollobás and O. Riordan, Mathematical Results on Scale-free Random Graphs, in Handbook of Graphs and Networks, Wiley-VCH, Berlin, 2002.

[10] B. Bollobás and O. Riordan, The diameter of a scale-free random graph, Combinatorica, 4 (2004) 5-34.

[11] B. Bollobás and O. Riordan, Coupling scale free and classical random graphs, Internet Mathematics 1 (2004), no. 2, 215-225.

[12] B. Bollobás, O. Riordan, J. Spencer and G. Tusanády, The degree sequence of a scale-free random graph process, Random Structures and Algorithms 18 (2001) 279-290.

[13] A. Broder, R. Kumar, F. Maghoul, P. Raghavan, S. Rajagopalan, R. Stata, A. Tomkins, and J. Wiener, Graph structure in the web, Proc. of the 9th Intl. World Wide Web Conference (2002) 309-320.

[14] G. Buckley and D. Osthus, Popularity based random graph models leading to a scale-free degree distribution, Discrete Mathematics 282 (2004) 53-68. 
[15] F.R.K. Chung, L. Lu, and V. Vu, Eigenvalues of random power law graphs, Annals of Combinatorics 7 (2003) 21-33.

[16] F.R.K. Chung, L. Lu, and V. Vu, The spectra of random graphs with expected degrees, Proceedings of national Academy of Sciences 100 (2003) 6313-6318.

[17] C. Cooper and A. M. Frieze, A General Model of Undirected Web Graphs, Random Structures and Algorithms, 22 (2003) 311-335.

[18] C. Cooper, A. M. Frieze, and J. Vera, Random deletions in a scale free random graph process, Internet Mathematics 1 (2004), 463-483.

[19] E. Drinea, M. Enachescu, and M. Mitzenmacher, Variations on Random Graph Models for the Web, Harvard Technical Report TR-06-01 (2001).

[20] E. Estrada, Spectral scaling and good expansion properties in complex networks, Europhysics Letters 73 (4) (2006) 649-655.

[21] P. Erdős and A. Rényi, On random graphs I, Publicationes Mathematicae Debrecen 6 (1959) 290-297.

[22] A. Fabrikant, E. Koutsoupias, and C. H. Papadimitriou, Heuristically Optimized Trade-Offs: A New Paradigm for Power Laws in the Internet, Proc. of 29th International Colloquium of Automata, Languages and Programming (2002) .

[23] M. Faloutsos, P. Faloutsos, and C. Faloutsos, On Power-law Relationships of the Internet Topology, ACM SIGCOMM Computer Communication Review 29 (1999) $251-262$.

[24] A. Flaxman, Expansion and lack thereof in randomly perturbed graphs, to appear in Proc. of the Web Algorithms Workshop (2006).

[25] A. Flaxman, A. M. Frieze and J. Vera, A Geometric Preferential Attachment Model of Networks, Internet Mathematics, to appear.

[26] J. Gómez-Gardeñes and Y. Moreno, Local versus global knowledge in the BarabásiAlbert scale-free network model, Physical Review E 69 (2004) 037103.

[27] B. Hayes, Graph theory in practice: Part II, American Scientist 88 (2000) 104-109.

[28] J. M. Kleinberg, R. Kumar, P. Raghavan, S. Rajagopalan, and A. S. Tomkins, The Web as a Graph: Measurements, Models and Methods, Proc. of the 5th Annual Intl. Conf. on Combinatorics and Computing (COCOON) (1999).

[29] R. Kumar, P. Raghavan, S. Rajagopalan, D. Sivakumar, A. Tomkins, and E. Upfal, Stochastic Models for the Web Graph, Proc. IEEE Symposium on Foundations of Computer Science (2000) 57.

[30] R. Kumar, P. Raghavan, S. Rajagopalan, D. Sivakumar, A. Tomkins, and E. Upfal, The Web as a Graph, Proc. 19th ACM SIGACT-SIGMOD-AIGART Symp. Principles of Database Systems (PODS) (2000) 1-10. 
[31] L. Li, D. Alderson, J. C. Doyle, W. Willinger, Towards a Theory of Scale-Free Graphs: Definition, Properties, and Implications, Internet Mathematics Vol. 2, No. $4,431-523$.

[32] M. Mihail, Complex networks: Connectivity and Functionality, an invited talk given at the NSF Workshop on the Theory of Networked Computation, Berkeley, March 15-16, 2006.

http://www.cc.gatech.edu/fac/Milena.Mihail/D.vita/icsi.ppt

[33] R. Kumar, P. Raghavan, S. Rajagopalan, and A. Tomkins, Trawling the Web for emerging cyber-communities, Computer Networks 31 (1999) 1481-1493.

[34] C. J. H. McDiarmid, Concentration, in Probabilistic methods in algorithmic discrete mathematics, (1998) 195-248.

[35] M. Mihail and C. H. Papadimitriou, On the Eigenvalue Power Law, Proc. of the 6th International Workshop on Randomization and Approximation Techniques (2002) 254-262.

[36] M. Mihail, C. H. Papadimitriou, and A. Saberi, On Certain Connectivity Properties of the Internet Topology, Proc. IEEE Symposium on Foundations of Computer Science (2003) 28.

[37] M. Mitzenmacher, A brief history of generative models for power law and lognormal distributions, Internet Mathematics 1 (2004), no. 2, 226-251.

[38] M. Newman, A.-L. Barabási, D. J. Watts, The Structure and Dynamics of Networks, Princeton University Press (2006).

[39] M. D. Penrose, Random Geometric Graphs, Oxford University Press (2003).

[40] H. A. Simon, On a class of skew distribution functions, Biometrika 42 (1955) 425440.

[41] R. van der Hofstad, Random Graphs and Complex Networks, unpublished manuscript (2007).

[42] D. J. Watts, Small Worlds: The Dynamics of Networks between Order and Randomness, Princeton: Princeton University Press (1999).

[43] G. Yule, A mathematical theory of evolution based on the conclusions of Dr. J.C. Willis, Philosophical Transactions of the Royal Society of London (Series B) 213 (1925) 21-87. 